Cahiers de recherches médiévales

Journal of medieval studies

16 | 2008

La réception d'Isidore de Séville durant le Moyen Âge tardif $\left(\left.X I\right|^{e}-X V^{e}\right.$ s.)

\title{
Les traductions françaises d'Isidore de Séville au Moyen Âge
}

Frédéric Duval

\section{CpenEdition}

\section{Journals}

Édition électronique

URL : https://journals.openedition.org/crm/10732

DOI : $10.4000 / \mathrm{crm} .10732$

ISSN : 1955-2424

Éditeur

Honoré Champion

Édition imprimée

Date de publication : 10 décembre 2008

Pagination : 93-105

ISSN : 1272-9752

Référence électronique

Frédéric Duval, "Les traductions françaises d'Isidore de Séville au Moyen Âge », Cahiers de recherches médiévales [En ligne], 16 | 2008, mis en ligne le 15 décembre 2011, consulté le 15 décembre 2022. URL : http://journals.openedition.org/crm/10732 ; DOI : https://doi.org/10.4000/crm.10732 


\title{
酷M
}

\section{Les traductions françaises d'Isidore de Séville au Moyen Âge}

\author{
Abstract: Despite its impressive fortune in Latin, the work of Isidore of Seville was only \\ rarely translated in medieval French. This article intends to identify and describe briefly all \\ the French translations : two of the Synonyma and a French version of the Monita (a centon \\ of the Synonyma) as well as a translation of the Chronicle.
}

Résumé : Malgré son impressionnante fortune latine, l'œuvre d'Isidore de Séville n'a fait l'objet que de très rares traductions en français médiéval. Cet article entend toutes les recenser. Il présente rapidement deux traductions des Synonyma et une version française des Monita (un centon des Synonyma) ainsi qu'une traduction de la Chronique.

Malgré son impressionnante fortune latine, l'œuvre d'Isidore de Séville n'a fait l'objet que de très rares traductions en français médiéval. Il est vrai que la théologie passe peu en langue vulgaire et que les Étymologies, le texte le plus copié d'Isidore, étaient difficilement traduisibles puisqu'elles reposaient sur l'examen de formes latines. Les textes qui ont été traduits devaient pouvoir s'adresser directement à des laïcs : tel est le cas à la fois des Synonymes, qui dispensent des conseils moraux essentiellement pratiques et peuvent aider à préparer un examen de conscience, mais aussi de la Chronique. Aucune des traductions françaises d'Isidore n'a connu le succès et on n'en a jamais conservé plus d'un manuscrit unique. C'est sans doute ce qui explique qu'elles aient été jusqu'ici si mal inventoriées. Ainsi, le répertoire de Paul Chavy ne recense qu'une traduction des Étymologies qui n'a jamais existé ${ }^{1}$. Il n'a donc pas paru inutile de présenter très brièvement, en nous appuyant sur les récentes éditions de la Chronique et des Synonymes ${ }^{2}$, l'ensemble des traductions repérées à ce jour.

\footnotetext{
${ }^{1}$ P. Chavy, Traducteurs d'autrefois : Moyen Âge et Renaissance, Dictionnaire des traducteurs et de la littérature traduite en ancien et moyen français (842-1600), Paris-Genève, Champion-Slatkine, 1988, t. I, p. 741-742.

${ }^{2}$ J. Elfassi, Les Synonyma d'Isidore de Séville : édition critique et histoire du texte, Paris, 2001 (thèse de l'EPHE-IV ${ }^{\mathrm{e}}$ Section). J. Elfassi a publié une partie de ses recherches sur la diffusion des Synonyma dans «Trois aspects inattendus de la postérité des Synonyma d'Isidore de Séville : les prières, les textes hagiographiques et les collections canoniques », Revue d'histoire des textes, n.s. 1, 2006, p. 109-152. Pour la Chronique, voir Isidori Hispalensis Chronica, éd. J. C. Martín, Turnhout, Brepols, 2003 (Corpus christianorum, Series Latina CXII).
}

Cahiers de Recherches Médiévales, 16, 2008 


\section{Les traductions des Synonyma}

\section{A) TRADUCTION LORRAINE ANONYME DU XII ${ }^{\mathrm{E}}$ SIECLE}

La plus ancienne traduction d'Isidore attribue le texte des Synonyma à saint Ambroise (Incipit dialogus beati Ambrosii anime conquerentis et rationis consolantis [rubriqué] f. 48). Son texte-source, reproduit dans le manuscrit, appartient, selon Jacques Elfassi « à la recension $\Phi$, et plus précisément elle paraît se rattacher à la famille $\mathrm{CRV}$, dont elle présente cinq variantes significatives ». Le manuscrit fait suivre immédiatement chaque paragraphe latin de sa version française, du moins jusqu'à II.31 (errores detrahentium calca, contumelias detrahentium patientia supera), passage où s'interrompt la traduction. L'interruption correspond à un changement de main dans le manuscrit, qui a conduit sans doute un peu vite $\mathrm{F}$. Bonnardot à identifier le traducteur avec le copiste de la première partie des Synonyma. La fin du texte latin a été transcrite par le copiste qui s'est chargé des deux textes suivants.

La traduction peut être datée du XII ${ }^{\mathrm{e}}$ siècle, comme le manuscrit qui nous la conserve $^{3}$. Elle suit fidèlement le texte latin, même si quelques phrases jugées trop redondantes sont parfois omises. Elle se caractérise par une «scripta » lorraine très marquée. Étudiée à la suite de son édition par F. Bonnardot, la langue de la traduction, probablement originaire d'un monastère vosgien, mériterait un nouvel examen approfondi.

\section{Incipits et explicits}

Incipit du prologue d'Isidore f. 48b : Ad mes mains est venuz novelement uns luvres, li ques est diz Sinonimes...

Incipit du texte f. 48b : M'anime est en anguise, et mes espiriz est chaufez, et mes curs est periliz..

Explicit du texte f. $65 \mathrm{~b}$ : reprove despisant les reproches des gas, vaint les errors finnant, et sormunte par patience les ledanges des mausdissanz.

\section{Description du manuscrit Épinal, bibliothèque municipale, ms 58}

a) Description matérielle

75 f. parchemin; 211 x $145 \mathrm{~mm}$; recueil confectionné à date ancienne à partir de deux manuscrits. Ms I : 47 f. (f. $1-47 \mathrm{v})$; début du XIII ${ }^{\mathrm{e}}$ siècle; copié à longues lignes à l'exception de textes adventices; f. 46v blanc. Ms II : 28 f. (f. 48$75 \mathrm{v}) ; 2^{\mathrm{e}}$ moitié du XII ${ }^{\mathrm{e}}$ siècle ; Lorraine ; copié sur 2 colonnes; dessins à l'encre pour illustrer chaque paragraphe du bestiaire. Provenance du recueil : Saint-Martin d'Épinal (cf. mention du XV ${ }^{\mathrm{e}}$ s., f. 75v).

\footnotetext{
${ }^{3} \mathrm{~B}$. Woledge et I. Short, «Liste provisoire de manuscrits du XII ${ }^{\mathrm{e}}$ siècle contenant des textes en langue française », Romania, 102, 1981, p. 5.
} 
b) Description du contenu

$\mathrm{I}^{\mathrm{re}}$ partie

1. Jean Beleth, Summa de ecclesiasticis officiis précédée d'une table analogique de $5 \mathrm{f}$. et demi non paginés, ajoutés en tête du manuscrit peu après la copie, f. $1-45 \mathrm{v}$

2. Marbode, Liber de gemmis (fragment de 53 vers), f. 46a-b

3. Liste des sept dons d'Adam (en latin), f. 46b

4. Compilation de préceptes liturgiques extraits de la Summa de ecclesisaticis officiis de Jean Beleth, f. 47-47v

$\mathrm{II}^{\mathrm{e}}$ partie

1. Isidore de Séville, Synonyma, texte latin et traduction lorraine anonyme partielle [titre ancien : Incipit dialogus beati Ambrosii anime conquerentis et rationis consolantis [rubriqué] f. 48a], f. 48a-70b (texte latin accompagné de la traduction française, f. $48 \mathrm{a}-65 \mathrm{~b}$; texte latin seul, f. $65 \mathrm{c}-70 \mathrm{~b}$ )

2. Hugues de Saint-Victor, De sacramentis christianae fidei (extraits) (inc. In Ecclesia duo sunt principalia sacramenta quibus homo et animal liberatur et bonis impletur: per sacramentum baptismatis mundamur a viciis, per sacramentum altaris implemur bonis), f. 70b-d

3. Dicta Johannis Chrysostomi de naturis bestiarum, f. 70d-75b

Bibliographie : Catalogue général des manuscrits des bibliothèques publiques de France, Paris, Plon, Nourrit et Cie, coll. in-4, t. III, 1861, p. 422 - F. Bonnardot, «Dialogus anime conquerentis et rationis consolantis: Traduction en dialecte lorrain du XII ${ }^{\mathrm{e}}$ siècle », Romania, 5, 1876, p. 269-332 (corrigenda par l'éditeur dans Romania, 6, 1877, p. 141-143, par W. Förster dans Zeitschrift für romanische Philologie, 1, 1877, p. 397-402, par. H. Suchier, ibid., p. 556-558 et par A. Tobler, ibid., p. 558-559) - B. Woledge et H. P. Clive, Répertoire des plus anciens textes en prose française depuis 842 jusqu'aux premières années du XIII siècle, Genève, Droz, 1964 (Publications romanes et françaises 79), p. $69 \mathrm{n}^{\circ} 18$ - Jean Beleth, Summa de ecclesiasticis officiis, éd. H. Douteil, Turnhout, Brepols, 1976 (Corpus christianorum, Continuatio mediaevalis 41), p. 110*-112* [notice détaillée du ms] notices inédites du ms aux sections latine et romane de l'IRHT - J. Elfassi, Les Synonyma d'Isidore de Séville, thèse citée, p. 126-127.

\section{B) TRADUCTION DE ROBERT DU VAL (1461-1466)}

La traduction des Synonyma que Robert du Val rédigea à l'attention de Jean de Montauban est le seul texte qu'il nous ait laissé. Si l'on s'appuie sur la dédicace, elle fut composée entre le 8 octobre 1461 (date de la nomination de Jean de Montauban à l'office d'amiral de France) et mai 1466 (date de sa mort) ${ }^{4}$. Le traducteur s'en tient au programme qu'il s'est fixé dans le prologue: sa version, fidèle au texte latin (recension $\Phi$, d'après J. Elfassi), ne présente ni ajout ni omission

${ }^{4}$ Voir U. Chevalier, Répertoire des sources historiques du Moyen Age, Paris, Société bibliographique, 1907, col. 2446; P Anselme, Histoire généalogique et chronologique de la Maison de France [...], Paris, 1733, t. VII, p. 856-857. 
remarquable. Nous ignorons presque tout de Robert du Val, que l'on peut identifier avec «maistre Robbert $d u$ Val, bachelier en theologie», au service du cardinal Balue en 1469, au moment où la bibliothèque du prélat fut saisie ${ }^{5}$. On sait, en effet, qu'au moment de la disgrâce du cardinal, Robert du Val avait entrepris la copie d'une traduction latine d'Appien (aujourd'hui ms Paris, Bibl. nat. de Fr., lat. 5785) et d'un Tite-Live (aujourd'hui Tours, bibl. mun. 984), qu'il acheva tous deux à la demande du roi. Les trois colophons du manuscrit de Tours, où Robert du Val se déclare natif de Rouen, comme dans la préface à la traduction des Synonyma, prouvent qu'il s'agit d'une seule et même personne ${ }^{6}$. Sa compétence théologique et son activité de copiste de textes latins confirment cette hypothèse. D'ailleurs, il n'est pas impossible que le traducteur se soit servi pour réaliser son travail de l'exemplaire latin des Synonyma conservé dans la bibliothèque du cardinal Balue ( ${ }^{\circ} 79$ de l'inventaire: "Ung petit livre en parchemin, contenant les Sinonimes Ysidiore; Indicuntur ») ${ }^{7}$. Même si l'écriture des luxueux manuscrits d'Appien et de Tite-Live a peu de points communs avec la cursive employée dans la traduction des Synonyma, plusieurs indices pourraient accréditer l'idée que l'unique et modeste manuscrit qui nous conserve cette traduction est autographe: le texte a été attentivement relu par le copiste qui a corrigé à plusieurs reprises des passages où l'on ne décèle pas de faute de copie évidente, sinon une seule dans le prologue. Ces corrections prennent la forme de ratures dans une encre rouge qui a également servi à souligner quelques titres de paragraphe; parfois, les mots barrés s'accompagnent d'ajouts interlinéaires.

\section{Incipits et explicits}

Incipit du prologue du traducteur f. 1: A tresnoble, trespuissant et excellent seigneur monseigneur de Montauban amiral de France, Robert du Val natif de Rouen...

Incipit du prologue d'Isidore f. 1: Le prologue de Ysidore | Nagueres est venu en mes mains ung petit livret appellé Synonimes conposé par Cicero dit Tulle...

Incipit du texte f. 2: L'omme "Anima mea"| Mon ame est contrainte par tristece par quoy mon esperit s'eschaufe et mon ceur se varie par diversses penssees...

Explicit du texte f. $42 \mathrm{v}$ : Il n'est au monde chose qui me soit plus chere que toy, qui me soit plus doulce. Tu me plais mieux que ma propre vie.

\footnotetext{
5 L. Delisle, Le cabinet des manuscrits de la Bibliothèque impériale, Paris, Imprimerie impériale, 1868, t. I, p. 79-83.

${ }^{6}$ Vicesimus tercius scriptus in domo domini cardinalis Andegavensis per Robertum suum familiarem Rothomago natum f. 154d ; Titi Livi xxiiii. per Robertum Rothomagensem finitur in domo cardinalis Andegavensis f. 165b; Titi Livi ab urbe condita liber .xxv. explicit in familia domini cardinalis Andeg. per Robertum de Valle Rothomagensem f. 174b.

${ }^{7}$ L. Delisle, Le cabinet des manuscrits... op. cit., p. 82.
} 


\section{fr. 2424}

Notice sommaire du manuscrit Paris, Bibliothèque nationale de France,

a) Description matérielle

$42 \mathrm{f}$. papier [filigrane : Licorne (Briquet 10005)] ; 217 x $145 \mathrm{~mm}$ (justification $140 \mathrm{x}$ $100 \mathrm{~mm}$ ); après 1460 (entre 1461 et 1466 si cette copie est bien celle qui a appartenu à Jean de Montauban); France ; copié à longues lignes par une seule main dans une écriture cursive peu homogène du $\mathrm{XV}^{\mathrm{e}}$ siècle, peut-être celle du traducteur Robert du Val (si l'on admet l'hypothèse d'un manuscrit autographe). Le texte a été corrigé de la main du copiste : des ratures à l'encre rouge qui a servi à souligner les titres sont parfois accompagnées de corrections intermarginales. Le décor se limite à des titres soulignés en rouge et à des lettres peintes hautes de deux lignes, alternativement en bleu et en rouge.

b) Description du contenu

1. Traduction des Synonyma d'Isidore de Séville par Robert du Val, f. 1-42v [titres anciens: Les synonimes de Ysidore f. 1; Cy est la fin dez synonymes de Ysidore translaté au bon desire de tresnoble et excellent seigneur monseigneur de Montauban, amiral de France, par maistre Robert du Val, natif de Rouen f. 42v] : prologue du traducteur f. 1-1v; prologue de l'auteur f. 1v; traduction f. 2-42v.

Sont indiqués ci-dessous pour chaque livre les titres de la traduction ainsi que le paragraphe des Synonyma dont ils sont suivis :

Livre I : L'omme "Anima mea» f. 2 (I.5); Raison "Quid tantum diffidis animo » f. 6 (1.22); Le homme «Qualiter » f. 6 (1.23); Raison f. 6v (1.24); L'omme f. 9v (1.39); Rayson f. 10 (1.40); L'omme f. 10 (1.41); Rayson f. 10 (1.42); L'omme f. 10 (1.43); Rayson f. 10v (1.46); L'omme f. 12 (1.52); Raison f. 12 (1.53); L'omme f. 12v (1.54); Rayson f. 12v (1.56); L'omme f. 13 (1.57); Raison f. $13 \mathrm{v}(1.58) ;$ 'omme f. 13v (1.62); Raison " Commota sunt » f. 17 (1.63)

Livre II : "Queso te anima» f. 18 (2.1); Raison f. 18 (2.2); De foy «Serva rectam» f. 18 (2.3); De pensee "Cor tuum» f. 18v (2.5); De fornicacion «Nulla jam » f. 19v (2.8); De humilité « Jam si» f. 22 (2.19); De son opposite come de orgueil et vantance "Cave autem» f. 22 (2.21); De contricion et penitance «Semper luges» f. 23 (2.24); De paour « Nulla te» f. 23 (2.25); De maladies «In infirmitatibus » f. 23v (2.26); De prosperité et adversité «Si prosperitas» f. 23v (2.27); De ire "Si prevenerit » f. 24v (2.30); Comme tu dois surmonter injure qui te est faicte «Quamvis quisque 》 f. 24v (2.32); De pardon «Petenti » f. 26 (2.35); De euvre "Quid zeli» f. 26v (2.37); De paix «Pacem ama» f. 26v (2.38); De compassion «Si ceciderit » f. 27 (2.39); Ensuir lez bons «In omnibus actionibus » f. $27 \mathrm{v}$ (2.41); De vaine gloire "Cave autem vanam etc." f. 28 (2.42); De conversation "Nec te adjungas» f. 28v (2.43); De parole "Claude aures » f. 29 (2.44); De detraction "Abscinde etiam» f. 30v (2.50); De curiosité " Quod autem ad te» f. 31 (2.52); De mentir et decepvoir «Omne quoque» f. 31v (2.53); De jurement et promesse «Proibe etiam » f. 32 (2.56); De pensee «Verbum iniqum»f. $32 \mathrm{v}(2.59)$; De tristece eviter «Nemo potest » f. 33 (2.61); De requerir conseil et l'aide de Dieu «Consilium» f. 33v (2.62); De confesser ses maulx "Vicia cordis » f. 33v (2.62); De sapience et doctrine "Nichil sapiencia» f. 34v (2.65); De reverence «Venerare» (2.73); Come se doit avoir le seigneur a ses serviteurs « $A$ 
subditis »f. 36v (2.76); De justice "Quod tibi» f. 37v (2.81); De humilité «In summo honore » f. 38v (2.87); De richesses «Divitie» f. 39v (2.91); De omosne "Contempne » f. 40v (2.95); Raison f. 41v (2.100); L'omme f. 42 (2.101)

\section{Édition de la dédicace, des prologues du traducteur et de l'auteur}

A tres noble, tres puissant et excellent seigneur monseigneur de Montauban admiral de France, Robert du Vaal natif de Rouen, honneur avec toute humilité et reverence.

Dit Seneque en son livre De moribus : Educatio et disciplina mores facit et id sapit quisque quod didicit ${ }^{8}$.

Donc vostre noblesse qui desire en soy et en ses subjetz avoir bonnes meurs m'a commandé a translater ce livre de latin en françois qui en soy contient bonne discipline, car il introduit l'omme qui pour ses pechés et les miseres de ce monde lamente jusques au desespoir, et Raison qui le conforte et remet en esperance. Aprés, luy baille enseignemens par lezquelz il se gardera de renchoir, evitera les temptacions et concupiscences et gardera la voie de bien vivre par laquelle ira a souveraine joie. Aucuns jugeront que il y ait superfluité de langage s'il ne considerent que Isidore ne vouloit mie a chascune oroison bailler sa sentence mais plusieurs convenir en une significacion qui fait dificulté a translater pour ce que langage françois n'est mie sy ample que latin et ne peult on trouver tant de motz françois synonimes que de latins. Pourquoy, consideré auxi le temps que j’ay, en bref submetz ceste euvre a la correction de vostre seigneurie et dez aultres lysans. [f. $1 \mathrm{v}$ ] Et se aucuns on en treuve, ce qui est orné en l'ung, souvent ne l'est mie en l'autre, pour ce que il est moult difficille que les couleurs et ornemens de rethorique qui sont en ung langage soient tousjours gardés en l'autre, comme dit Jeronimmus in epistulis ${ }^{9}$.

Le prologue de Ysidore

Nagueres est venu en mes mains ung petit livret appellé Synonimes conposé par Cicero dit Tulle, duquel la forme amonnesta et esmeut mon courage de conpiler et composer le livre present tant a l'utillité de moy que des aultres qui seront en misere. Et en ce faisant, je n'ensuivray point du toult la maniere de Tulle, mes feray selon ma propre invention, car Tulle fist seulement noms et dictions synonimes sans quelques sentence, et je feray oroisons synonimes qui auront en soi bonne sentence. Icelle donc lis et estudie volentiers, et quant tu seras en adversité, tu te pourras par icelle congnoistre et les causes d'icelle adversité. Et auxi congnoitras et entendras que quelconques afflictions tu seuffres en ce monde, il te seront tres justement retribuees en l'autre advenir. Et seront introduites deulx personnes : l'une de l'omme qui pleure, l'autre de Raison qui l'amonneste et conforte.

Bibliographie : Bibliothèque impériale, départements des manuscrits, Catalogue des manuscrits français. Ancien fonds, Paris, Leroux, t. I, 1868, p. 417 - notice du

\footnotetext{
${ }^{8}$ didiscit dans le manuscrit. - Cette citation est tirée du $\S 2$ du De moribus, un recueil anonyme de proverbes attribué au Moyen Age à Sénèque.

${ }^{9}$ Allusion à la préface de la Vulgate par saint Jérôme.
} 
ms par Fr. Girard (1972) conservée à la section romane de l'IRHT - J. Elfassi, Les Synonyma d'Isidore de Séville, thèse citée, p. 127.

\section{C) LES ADMONNESTEMENS PRINS DES PAROLES DU SAINCT YSIDOIRE, TRADUCTION ANONYME DES MONITA, CENTON DES SYNONYMA}

La traduction anonyme des Monita date, d'après sa langue, du $\mathrm{XV}^{\mathrm{e}}$ siècle. Le traducteur a pris d'importantes libertés avec le texte latin qu'il n'hésite pas à remanier, tantôt en le développant, tantôt en supprimant des membres de phrase, voire des phrases entières. Le principe stylistique des synonymes n'a pas été compris ni respecté, si bien que le texte est souvent retouché pour éviter les «redites». La version française se signale par une langue moins imagée que son texte-source et par l'ajout de phrases conclusives à la fin des paragraphes.

Il n'est pas impossible que cette traduction des Monita ait été spécialement conçue dans le cadre de la réalisation de l'unique manuscrit qui nous l'a conservée. Ce manuscrit de luxe, manifestement destiné à un laïc, devait servir à préparer et à suivre les messes dominicales. Il s'agit en fait d'un lectionnaire en français, qui présente les lectures des épîtres et évangiles selon l'ordre du calendrier liturgique. Une introduction d'une petite vingtaine de feuillets précède la partie lectionnaire proprement dite. On y a copié bout à bout, sans un seul saut de ligne, de courts textes permettant au fidèle de se préparer à la célébration. La traduction des Monita, placée en tête de l'introduction, est nettement orientée vers la préparation de la messe, comme le prouve le long développement qui suit la traduction du premier paragraphe (voir édition infra). Les différents extraits composant l'introduction ne sont pas nettement séparés par la mise en page et le lecteur serait bien en peine de comprendre où prend fin la traduction d'Isidore sans avoir à sa disposition le texte latin. Seule la table donne quelques clefs pour comprendre la composition de cette introduction-compilation qui s'achève par un titre de fin bien imprécis : «Finitur liber documentor Ysodori | Finitur cronice Romanorum | Ici prendra l'an exemplaires bons et utiles » (f. 21a).

\section{Incipits et explicits}

Incipit f. 4b: Homme, metz paine comment tu te puisse congnoistre. Saches quelle chose tu es et en quelle chose tu es engendré, a quoy tu es né, pour quoy tu es fait, par quelle condicion tu es venu avant, a quoy tu es ordené au siecle, remembre toy de ta condicion...

Explicit f. 8d: ... ne fais pas ton don pour avoir humaine louenge, ne pour temporelle oppinion mais pour l'amour de Dieu et pour avoir vie pardurable.

\section{fr. 402}

Notice sommaire du manuscrit Paris, Bibliothèque nationale de France,

a) Description matérielle

168 f. parchemin, précédés d'1 f. de garde papier moderne et de $3 \mathrm{f}$. de garde parch. et suivis de $3 \mathrm{f}$. de garde parchemin ; 355 x $255 \mathrm{~mm}$ (justification à l'encre 
rouge $233 \times 157 \mathrm{~mm})$; $2^{\mathrm{e}}$ moitié du $\mathrm{XV}^{\mathrm{e}}$ siècle; Tours ?; f. $3 \mathrm{v}$ blanc ; copié sur 2 colonnes par une seule main. Décoration: une grande miniature $(200 \times 153 \mathrm{~mm})$ avec encadrement représentant Dieu en majesté entouré des symboles des quatre évangélistes (f. 4). D'après la base Mandragore, cette miniature daterait de la seconde moitié du $\mathrm{XV}^{\mathrm{e}} \mathrm{s}$. et aurait été réalisée à Tours ${ }^{10}$. Chaque paragraphe s'ouvre par une lettre champie en or sur fond bleu ou grenat avec décor filigrané et jeu d'alternance des couleurs. Des rehauts de jaunes viennent marquer les articulations du discours.

b) Description du contenu

\section{Table}

1. Table du manuscrit, f. 1a-3b [Cy commence la table du livre des espitres et evangilles translatee de latin en françoys avecques aultres ensaignemens mis et aposés au commencement de cest livre [rubriqué] f. 1a].

f. $3 \mathrm{v}$ blanc

\section{Introduction}

2. Traduction anonyme des Monita, f. 4a-8d [titre ancien: Cy ensuivent les admonnestemens prins des paroles $d u$ sainct Ysidoire pour ensaigner l'omme comment il puisse peché eviter et se puisse enfermer en bien. Premierement de la consideracion de soy mesmes [rubriqué] f. 4a].

Sont indiquées ci-dessous les rubriques du manuscrit suivies du ou des paragraphes des Synonyma auxquelles elles correspondent:

Premierement de la consideracion de soy mesme (2.2); Pour contrarier a mauvaises cogitacions (2.5-7); De attraire a soy chasteté $(2.8)$; De soy mettre en oroison (2.10-13); De apprendre a jeuner (2.14-15); De regarder chastement (2.16-17); De donner maniere de fuir les femmes (2.17); De soy mettre en labour (2.18-19); De entendre a Dieu (2.19); De actraire humilité (2.20-21, 23, 87); De tenir l'ame en bonnes pensees (2.26-29); De avoir pascience (2.30-34); De attraire paix (2.38-39); D'avoir compassion (2.39-40); De ensuivir les bons (2.41); De despriser louenge (2.42); De honneste conversacion (2.43); De bonne compaignie (2.43-44); De honnestement escouter (2.44-45); De garder sa bouche (2.45-49); De soy garder de detraction (2.50-52); De fuir mensonges (2.53-56); De rendre son veu (2.57-58); Comment toutes choses sont davant Dieu apertes (2.59-60); De acquerir bonne conscience (2.61); Come tout bien doit estre attribué a Dieu (2.62); Comme vertus et bonnes operacions doivent estre celees (2.62); De soy ordonner a confession (2.62); De soy pourpenser avant le fait (2.63); De considerer qu'est sapience (2.65-66); De apprendre doctrine (2.67-70); De fuir curiosité inutille (2.71-73); De obedience et reverence $(2.73-74,88,76)$; De gouverner seignourie (2.76-79, 81-86); De fuir le monde (2.92, 95); De faire aulmonnes (2.96-98).

3. Des proprietés des .vii. pechés mortelz [rubriqué], f. $8 \mathrm{~d}$

4. Du jugement et des ensaignemens de Salomon du livre de Sapience [rubriqué], f. 8d-16a (inc.: Ou tamps que Salomon filz du roy David fut roy de Jherusalem, Dieu luy monstra moult grant amour, car on list en l'Escripture qu'il

${ }^{10}$ La base Mandragore est consultable à l'adresse suivante : <mandragore.bnf.fr> . 
fut sainctifié dés son enffance... expl. : ... mais celui qui vient petit a petit durera longuement)

5. La signifiance comment on doit penser en la messe [rubriqué], f. 16a-18d (inc. : Premierement quant on oyt sonner la messe, on doit penser que ce sont les messagers de Dieu... expl. : et que je puisse parvenir en corps et en ame en vostre saincte gloire que vous avés promise a vos amis, laquelle vous nous voulés octroier par vostre saincte misericorde. Amen)

6. [Chronologie universelle], f. 18d-20b (inc. : Nostre seigneur forma Adam depuis ce qu'il eut fait le ciel et la terre... expl. : En celui an meismes occirent les Frisons le roy Dais). La rupture avec le texte précédent n'est pas signalée par une rubrique. Seule une initiale champie haute de trois lignes, alors que les précédentes ne l'étaient que de deux, signale une articulation pourtant annoncée dans la table.

7. [Les saintz lieux de Jherusalem], f. 20b-21a (inc. : Cy poués vous sçavoir les saintz lieux de Jherusalem. En Jherusalem a ung sainct lieu couvert... expl. : La voult Abraham sacrifier a Dieu et Dieu parla a Abraham et lui dit qu'il ne sacrifia pas son filz mes ung mouton qui estoit davant lui). Aucune rupture avec ce qui précède, sinon par une initiale champie haute de 2 lignes.

\section{Lectionnaire}

8. Lectionnaire présentant les lectures de la messe (épîtres et évangiles) dans l'ordre de l'année liturgique, f. 21b-167a (incipit f. 21b : Cy commence la premiere epistre du premier dimence de l'advent nostre Seigneur : saint Paoul ad Romanos [rubriqué] | Beaulz freres, sachés qu'il est heure de soy lever de dormir maintenant)

\section{Extrait}

Édition de la traduction du premier paragraphe des Monita (= Synonyma 2.2) et du long développement qui la suit

[f. 4b] Homme, metz paine comment tu te puisse congnoistre. Saches quelle chose tu es et en quelle chose tu es engendré, a quoy tu es né, pour quoy tu es fait, par quelle condicion tu es venu avant, a quoy tu es ordené au siecle, remembre toy de ta condicion, regarde que Dieu t'a fait et [f. 4c] quel ton createur t'a establi. 9 Premierement je dy que homme doibt mectre paine de soy congnoistre, car c'est voye de Dieu de soy cognoistre, car nous fusmes fais a sa samblance et a son ymage, car ung ymage si fait proprement cognoistre celui pour qui il est fait, come les ymages de sainct Pierre et de sainct Pol. Ainsi si bien nous congnoissons que nous sommes ymage de Dieu, nous porrons le congnoistre en aucune maniere, laquelle chose nous est neccessaire selon ce qu'il est escript Hec est vita eterna. I Nul ne peult avoir vie pardurable s'il ne congnoist Dieu le pere, Jhesuscrist qu'il a envoyé en terre pour nostre redemption; et ceulx qui ne estudient a avoir ceste congnoissance reprent saint $\mathrm{Be}[\mathrm{r}]$ nard, qui dit: "Moult de gens pensent sçavoir moult de choses pour estre reputés clers et pour avoir les promocions de cestui monde et les aultres regardent et jugent et eulx ne veulent regarder ne juger par lequel regard nous pouons acquerir le regard et la science de nostre savement et les promissions de paradis. Pour ce dit ung docteur: «Quid prodest... Que vault considerer les constellacions et les cours des estoilles, la disposicion des planectes et les vertus des herbes, la dignité des pierres precieuses et avoir ignorance de soy, non 
pas de soy seulement mais de son createur et de soy, qui trop nuit et empesche tout bien congnoistre? » Aprés il fault considerer quelle chose nous sommes. Tu es homme qui a la samblance de Dieu es fait; garde que celui ymage soit droittement selon la forme de Dieu. Deu est espirituel, non pas charnel. Quant est de soy, gardes que tu vives selon espiritualité et non pas selon charnalité. Et si tu dis que le filz de Dieu print nostre humanité, tu dis verité, mes il [f. 4d] ne print par la charnalité, la difformité ne la superfluité que nous avons, mais il print nostre nature quant a naturele condicion, circonscripte, toute desordenante, peccable qui vint par le premier peché. Dieu ne tient compte de convoitise mondaine ne des richesses temporelles ne de superfluité ; il nasquit d'une pouvre vierge et en povre lieu quant aux biens du monde et vesquit en povreté et print povre gent en sa compaignie. Pour ce doncques fut humble et debonaire, sicome il dit: "Discite a me, quia mitis sum et humilis corde». Il ne fust pas orguilleux ne ne quist grant beubans unde dicebat: "Regnum meum non est de hoc mundo ». Si tu es dont ymage de Dieu, fais que tu soyes paint en sa forme. Aprés je dis que tu es homme qui a corps et ame. L'ame vient de Dieu le pere Jhesuscrist par creacion, et le corps vient de terre et ordure par corrupcion. Le corps est corrumpable, car il n'est fors que viande a vers, qui sont pourriture et ordure. Or regarde comment la noble ame est perpetuelement acompaignie des tresglorieux anges de paradis. L'ame est la vie du corps : sans l'ame, le corps n'est que pourriture et ordure. Si regardes donques comment tu es composé. Aime mieulx tu doncques la partie qui mieulx vault, c'est l'ame? Pense doncques a garder l'ame mieulx que le corps, quiers premier la vie de l'ame. Et si demandois qu'est ce que la vie de l'ame, c'est la grace de Dieu, car selon saint Augustin, aussi comme l'ame est la vie du corps, ainsi est la grace de Dieu la vie de l'ame. Si tu quiers a soustenir l'ame affin que le corps vive, quiers premierement a avoir la grace de Dieu qui est la vie de l'ame. Soustiens doncques l'ame premierement. Le nourrissement de l'ame est predications, devocions et oroisons, bonnes parolles, bonnes [f. 5a] pensees ${ }^{11}$ et bonnes oeuvres. Regarde doncques de quelle chose tu es et de la principale chose use principalement. Aussy come tu es composé d'ame et de corps, tu es naiz a faire deux choses : l'operacion de l'ame et l'operacion du corps. En l'ame a .iii. puissances, lesquelles elle doit avoir, c'est assavoir entendement, voulenté et memoire : entendement pour discerner entre le bien et le mal, voulenté pour le bien faire et le mal haïr, memoire pour le bien retenir et le mal oublier. Or regarde doncques que tu aies ces .iii. operacions qui sont attribuees a l'ame. Le corps a cincq puissances, ce sont les cincq sens naturelz de quoy homme doit user et tellement le corps gouverner qu'il soit tousjours subget a l'ame et que ses operacions ne contredient point aux operacions de l'ame. Homme est nés affin que l'ame tende tousjours a Dieu par devocion et souspirs de dilection et que le corps soit tousjours occupé en labour de bonnes oeuvres et exercitacion corporelle pour reprimer la coincteté de la char et la rebellion et fuire occiosité qui est cause de tout mal. - Regarde aussi que tu es engendré et nés en peché enclin et disposé a peché. Et pource toutes choses qui esmouvent a peché tu dois fouir et eschever. Regarde aprés pourquoy tu es fait pour servir a Dieu. C'est belle chose de servir, aimer, honnourer, Dieu doubter, qui tant a fait de bien et de fuir, regnoier, despiter celuy qui tant t'a fait et veult tousjours faire mal, car Dieu descendit en terre

${ }^{11}$ bonnes pen pensees dans le ms. 
pour nous servir, non pas seulement pour nous servir qui devons ${ }^{12}$ estre ses serfs, mais pour nous racheter des dangers de la mort perpetuelle ou nous estions tous condampnés. Il nous a racheté de service dampnable. Nous ly devons dont faire service honnourable, car tout l'onneur que nous ${ }^{13}$ luy faisons vient de luy [f. 5b] et si nous est prouffitable. Il nous aima tant que pour nous il voult mourir. Or l'aimons dont pour vivre en sa gloire, car si nous l'aimons, nous ne mourrons pas. Il nous a tant honnourés que homme est et Dieu homme est. Ceste chose n'a il pas faicte aux anges. Il nous a tant honnourés que a la dextre de son pere nous a essaucié. Honnourons doncques en terre celuy qui tant nous honnoura es cielx. Ne portons donc pas plus de honneur a creature que a luy et ne aimons ne servons plus creature que Dieu et du tout nous mectons a faire et tenir ses commandemens fermement et ainsi lui ferons honneur et despit a l'ennemy et a nous proffit perpetuel. Donc nous sommes nés pour lui servir, car il a fait toutes choses pour nous. Or le servons donc et le monde nous servira. Si nous servons mauvaisement Dieu, le monde mal nous servira. Le monde nous doit .iii. choses pour lesquelles nous devons Dieu honnourer principalement et porter reverence. Pour ce dit Dieu aux aultres creatures quant il forma le monde qu'il fussent subgetz et obeissans a l'omme; et il est vrai car nous voyons de jour et de nuit le ciel, les estoilles et le souleil et les planectes qui y sont et aussi tous les quatres elemens qui nous monstrent service, amour et aliance, car toute leur bonté nous anticipons. Aussi Dieu a ordonné que le monde eut a nous cremour et adjoustance, et tant que devant le premier peché toute creature simple et corporele doubtoit et cremoit homme ne contre lui ne se osast prendre; dont puis que le monde estoit fait pour nous servir, nous portat honneur, amour et crainte, et nous a Dieu, de qui sommes creés pour luy servir. Nous devons donc a Dieu service, amour et aliance, honneur et reverence, crainte et doubtance. Et se ces choses nous rendissons a Dieu, le monde les nous rendist temporellement.

Bibliographie : P. Paris, Les manuscrits françois de la Bibliothèque du roi, Paris, Techener, 1836-1846, t. III, p. 367-368 - Bibliothèque impériale, départements des manuscrits, Catalogue des manuscrits français, Ancien fonds, t. I, Paris, Leroux, 1868, p. 38 - J. Elfassi, Les Synonyma d'Isidore de Séville, thèse citée, p. $774-$ J. Elfassi, «Los centones de los Synonyma de Isidoro de Sevilla », dans Actas do IV Congresso Internacional de Latim Medieval Hispânico (Lisboa, 12-15 de Outubro de 2005), éd. A. A. Nascimento et P. F. Alberto, Lisboa, Centro de Estudos Clássicos, 2006, p. 393-401, spéc. p. 398 - Mandragore <mandragore.bnf.fr>.

\section{Traduction de la Chronique}

Probablement exécuté en Italie du sud dans le deuxième quart du XIV siècle, le manuscrit Paris, Bibl. nat. de Fr., fr. 688 est un recueil de textes historiques traduits du latin au français par un traducteur anonyme à l'intention - et peut-être à la demande - du comte de Militrée, un noble italien qui n'a toujours pas été identifié. La précision des gloses géographiques ainsi que la langue, très marquée

\footnotetext{
${ }^{12}$ doivons dans le ms.

${ }^{13}$ que je luy dans le ms.
} 
par les dialectes de l'Italie méridionale, laissent à penser que le traducteur était un Italien du sud, peut-être originaire de Campanie (d'après N. Moreau).

Le manuscrit s'ouvre avec la traduction de la première rédaction de la Chronique d'Isidore de Séville, caractérisée par la référence à Sisebut à la fin du texte. Alors que le prologue d'Isidore n'est pas traduit, le traducteur annonce dans son propre prologue qu'il traduira littéralement (secont la lettre) et complétera, si possible, le texte latin ( $i$ adjondrai aucunes bones paroles de verité). Outre les gloses géographiques et lexicales, la plupart des additions portent sur la mythologie, l'histoire de Troie, les institutions religieuses et la Bible.

\section{Incipits et explicits}

Prologue

Voir édition infra

Texte

Incipit f. 1c: Nous trovons en la sainte escripture divine de la Bible que le Seignor Dieu, pere tout puissant, fist et forma et cria toutes chozes

Explicit f. 11b: Aiez en ton cuer et en ta pense la fin toe, maiz non pecheras, quar a chascun qui de cest monde passe, le monde est finé pour lui.

\section{Description du manuscrit Paris, BNF, fr. 688}

a) Description matérielle

$212 \mathrm{f}$. parchemin; $355 \times 250 \mathrm{~mm}$ (justification $240 \times 165 \mathrm{~mm}$ ); $2^{\mathrm{e}}$ quart du $\mathrm{XIV}^{\mathrm{e}}$ siècle; Italie du sud; copiés sur 2 colonnes par une seule main. Décoration: une grande miniature $(315 \times 195 \mathrm{~mm})$ à huit compartiments retraçant la création du monde, avec encadrement (f. 1) ; lettres champies (or sur fond alternativement bleu ou rouge); lettres historiées.

b) Description du contenu

1. Traduction anonyme de la Chronique d'Isidore de Séville, f. 1-11 [titres anciens: Ci se comence li livre de la cronique del vaillant evesque Ysodore, [f. 1c] le quel a fait exposition en aucune choze del premier livre de la Bible et aprés de mult croniques de ppapes et d'emperators et de mult autres estoires et bataillez et conquestes [rubriqué]; Ci se finist la cronique de Ysodore, la [quel] est escripte en vulgal fransoiz [rubriqué] f. 11b]

2. Traduction anonyme de l'Historia romana de Paul Diacre, f. 11c-72 $125 \mathrm{~d}$

3. Traduction anonyme de l'Historia Langobardorum de Paul Diacre, f. 72-

4. Traduction anonyme de l'Historia Normannorum d'Aimé du Mont-Cassin, f. $125 \mathrm{~d}-199 \mathrm{a}$

5. Traduction anonyme de l'Historia Sicula, f. 199a-212d

Édition du prologue du traducteur

$\mathrm{Ci}$ se comence le proheme de la translatation, la quel fait faire le seignor conte de Militree, etc. [rubriqué] 
Secont ce que nouz dit et raconte lo sage phylosofo, tout home naturalment desirre de savoir, et la raison si est ceste car toute choze covoite et desirre sa perfeccion. Mes il n'est nulle choze qui face l'ome plus parfait que science, quar par la science est home fait semblable a Dieu. Adonc l'omo doit desirrer et covoitier come pour sa perfeccion la science. Et toutes voiez savoir et science sont aquestees et seuez especialment par litterature. Et non portant toz les homes qui sont ne poënt pas estre si grans maistres en litterature qu'il puissent entendre la sentence de la letre et, pour ce, juste choze est que ceauz les quelz ne poënt parfaitement entendre la grammere par la quelle sont ordenéz et faiz [f. 1b] les livres, qu'il facent translater la lettre en alcune vulgal langue pour ce qu'il puissent savoir et entendre aucunes escriptures des quelles il ont delettation et volenté de savoir.

Et pour ces choze devant dite, plot et pensa monseignor conte de Militree qu'il feroit translater en vulgal la cronique de Ysidorre secont la lettre. Et pour ce qu'il set lire et entendre la lengue fransoize et s'en delitte, a fait translater par ordre secont la lettre en françois la devant ditte cronique, et especialement pour sa delettation et pour la delettation de ses amis. Mes pour la rayson de ce que aucune foiz plusors croniques parlent trop brief, je qui li livre escrive de letre en vulgal, se je puiz, i adjondrai aucunes bones paroles de verité.

Explicit prologus [rubriqué]

Bibliographie : P. Paris, Les manuscrits françois de la bibliothèque du roi [...], Paris, Techener, 1836-1848, t. V, p. 332-344 - F. Torraca, « Amato di Montecassino e il suo traduttore », Casinensia, 1, 1929, p. 161-170 [sur l'identité du traducteur] E. Sthamer, «Der Mönch Azzo von Montecassino », Sitzungsbericht der Preussischen Akademie der Wissenschaften, 1932, p. 670-677 [sur l'identité du traducteur] - Storia dei Normanni di Amato di Montecassino volgarizzata in antico francese, éd. V. De Bartholomaeis, Roma, Tipografia del Senato, 1935 (Fonti per la storia d'italia 76), p. Ixxxviii-xcv - M. D'Onofrio (dir.), Les Normands : peuple d'Europe 1030-1200, Paris, Flammarion, 1994, p. 380-381 [notice du manuscrit par M.-Th. Gousset] - M. Gasperoni et S. Maffei, « Considerazioni sul manoscritto f. fr. 688 della Biblioteca Nazionale di Parigi: l'Ystoire romane e l'Ystoire de li Longobart di Paolo Diacono », Francofonia, 30, 1996, p. 53-80 - S. Maffei, « Dall' 'Historia Langobardorum' all' 'Ystoire de li Longobart': tra fedeltà e innovazione », Le letterature romanze del Medioevo : testi, storia, intersezioni, Società italiana di filologia romanza, V Convegno Nazionale, Roma, 23-25 ottobre 1997, éd. A. Pioletti, Rubbettino, Soveria Mannelli, 2000 (Medioevo romanzo e orientale, Colloqui 5), p. 133-166- N. Moreau, Le manuscrit fr. 688 et son traducteur: édition et commentaire de la Chronique universelle et de l'Histoire romaine, thèse d'École des chartes, 2001 [édition, t. II, p. 4-30] ; cf. Positions des thèses soutenues par les élèves de la promotion de 2001 pour obtenir le diplôme d'archiviste paléographe, Paris, École des chartes, 2001, p. 127-133 - Isidori Hispalensis Chronica, éd. J. C. Martín, Turnhout, Brepols, 2003 (Corpus christianorum, Series Latina CXII) [particulièrement p. 55*, 221*-222*].

Frédéric Duval 